\title{
Comparative Study Between Peristomal Patches in Patients with Definitive Tracheostomy
}

\author{
Liset Lansaat ${ }^{1} \quad$ Bertram de Kleijn $^{2}$ Frans Hilgers ${ }^{1,3}$ \\ ${ }^{1}$ Department of Head and Neck Surgery and Oncology, Nederlands \\ Kanker Instituut - Antoni van Leeuwenhoek Ziekenhuis, \\ Amsterdam, The Netherlands \\ 2 Department of Otorhinolaryngology/Head and Neck Surgery, \\ Universitair Medisch Centrum Groningen, Groningen, The Netherlands \\ ${ }^{3}$ Institute of Phonetic Sciences, University of Amsterdan, \\ Amsterdan, The Netherlands \\ ${ }^{4}$ Department of Maxillofacial Surgery, Academic Medical Center, \\ University of Amsterdan, Amsterdan, The Netherlands
}

Bernard van der Laan ${ }^{2}$ Michiel van den Brekel ${ }^{1,3,4}$

Address for correspondence Liset Lansaat, MSc, Hoofd and Hals Oncologie and Chirurgie, Nederlands Kanker Instituut - Antoni van Leeuwenhoek Ziekenhuis, Plesmanlaan 121, 1066 CX Amsterdam, Noord-Holland 1006 BE, Netherlands (e-mail: I.lansaat@nki.nl).

Int Arch Otorhinolaryngol 2018;22:130-135.

\begin{abstract}
Introduction To prevent or diminish pulmonary problems in laryngectomized patients, continuous use of a heat and moisture exchanger (HME) is recommended. Therefore, automatic speaking valves are also often combined with an HME to enable hands-free speech. In order to keep these devices in place, most commonly, peristomal patches are used.

Objective This prospective clinical $2 \times 2$ crossover study aims at assessing the added value of a new patch for HME application, the Provox StabiliBase OptiDerm (SBO). The device combines the stable and conical base of the Provox StabiliBase with the skinfriendlier hydrocolloid Provox OptiDerm (OD) patch.

Methods Thirty-two laryngectomized patients were included in this multicenter study. Participants were asked to compare SBO to OD, and to the patch they normally use. The primary outcome measure was patient preference.

Results Overall, $60 \%$ of the participants had preference for their normally used patch,

Keywords

- laryngectomy

- rehabilitation

- patch

- patient preference $23 \%$ preferred the SBO and $17 \%$ indicated no preference. When comparing the SBO to the OD, $43 \%$ preferred the SBO, $40 \%$ the OD and $17 \%$ had no preference.

Conclusion Most patients preferred their normally used patch and SBO was favored by a subgroup. Provox StabiliBase OptiDerm seems to be a valuable addition to the existing patches and further increases patients' options for HME application.
\end{abstract}

\section{Introduction}

Total laryngectomy (TL) is still an indispensable treatment option for advanced larynx and hypopharynx cancer, for recurrent disease, and a dysfunctional larynx after prior (chemo) radiotherapy (C)RT. Total laryngectomy results in significant anatomical changes. The alimentary and respiratory tracts are separated and a definitive tracheostomy is created at the base of the neck. The main disadvantage of TL is the loss of upper airway and larynx functions. This leads to pulmonary problems, such as excessive coughing and mucus production, and loss of normal speech. ${ }^{1,2}$

To prevent or diminish pulmonary problems, continuous use of a heat and moisture exchanger (HME) has shown to be highly beneficial. ${ }^{3,4}$ Moreover, most automatic speaking valves (ASVs) presently are combined with an HME, so that during hands-free tracheoesophageal speech, airway protection and rehabilitation are also taken care of. ${ }^{5-7}$ Laryngectomized patients have several options to keep these devices in place depending on their personal situation. The most commonly received

February 15, 2017

accepted

May 13, 2017

published online

June 16, 2017
DOI https://doi.org/

$10.1055 / \mathrm{s}-0037-1603921$. ISSN 1809-9777.
Copyright $\odot 2018$ by Thieme Revinter

Publicações Ltda, Rio de Janeiro, Brazil
License terms

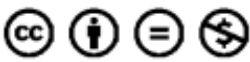


used device is a peristomal patch, which creates an airtight seal at the level of the tracheostoma and provides a placeholder for the HME and/or ASV. ${ }^{8}$

Currently, there is a wide variety of patches available to suit patients' personal needs, which is important to optimize compliance. ${ }^{8-10}$ Recently, the Provox StabiliBase (SB) was evaluated in a multicenter study. This patch provides a more stable and more anatomically shaped conical base compared with other patches. The study showed that the majority of patients preferred this new patch to their usual comparator, and its device life appeared to be significantly longer. Also, patients with a deep stoma reported the patch to be more comfortable. ${ }^{9}$

After its introduction, feedback from clinicians and patients revealed that some patients experienced skin irritation with the standard adhesive material of the SB. It was felt that these patients would benefit from a patch with the same stable and conical base as the SB, but with the more skin-friendly hydrocolloid adhesive already used in the Provox OptiDerm (OD). Therefore, the Provox StabiliBase OptiDerm (SBO) was developed. To test whether this stable conical hydrocolloid SBO patch is a valuable addition to the variety of peristomal adhesive options needed to suit more laryngectomized patients, this new patch was assessed in a $2 \times 2$ crossover prospective multicenter clinical trial.

\section{Methods}

This study was performed at two tertiary care cancer centers. Thirty-two laryngectomized patients were entered in the study, 16 from each center. Inclusion criteria were: 18 years or older, use of an HME, use of a voice prosthesis, minimum of 3 months after TL and/or postoperative (C)RT. Exclusion criteria were: patient is unable to use the SBO (due to anatomical irregularities that may interfere with the stable base of the patch), medical problems prohibiting the use of HME or patch, active recurrent or metastatic disease, patient is unable to understand the patient information and/or unable to give informed consent. Skin irritation, which varies between 9 and $40 \%$ among patients, ${ }^{4,10-12}$ was not a selection criterion. This provides us with two advantages, namely additional data on the extent of the skin irritation problem in this patient cohort, and prevention of selection bias. Moreover, it is likely that, if given more options and provided that there was no skin irritation, patients would primarily make their choice on the basis of the duration of the seal. In other words, by using an unselected patient cohort, we can get a better insight of the extent of the irritation problem and of the place of this new patch among the presently available options. The study was performed according to the protocol approved by the institutional review boards and took place between February and April of 2014. Signed informed consent was obtained from all patients. Patient characteristics are shown in - Table 1.

The SBO is manufactured by Atos Medical AB (Hörby, Sweden). The patch is shown in - Fig. 1. It is a single-use patch intended for laryngectomized patients. It is attached to the skin around the tracheostoma to provide a connection for HMEs and speaking valves. The SBO consists of a stable base, similar to that of the SB, but with a hydrocolloid adhesive. ${ }^{9}$ The patch is
Table 1 Patient characteristics

\begin{tabular}{|c|c|c|}
\hline Characteristics & Value & $\%$ \\
\hline \multicolumn{3}{|l|}{ Gender } \\
\hline Male & 27 & 84 \\
\hline Female & 5 & 16 \\
\hline Age at $T L$ & Mean 55.7 years (SD 9.4) & \\
\hline Age at entry & Mean 64.0 years $(48-82)$ & \\
\hline Post-TL & $\begin{array}{l}\text { Mean } 100.7 \text { months } \\
\text { (SD 77.9) }\end{array}$ & \\
\hline \multicolumn{3}{|l|}{$T L$} \\
\hline Standard & 28 & 88 \\
\hline+ Reconstruction & 3 & 9 \\
\hline Information missing & 1 & 3 \\
\hline \multicolumn{3}{|l|}{ Origin of tumor } \\
\hline Larynx & 30 & 94 \\
\hline Hypopharynx & 2 & 6 \\
\hline \multicolumn{3}{|l|}{ Indication of TL } \\
\hline Primary & 9 & 28 \\
\hline Salvage & 23 & 72 \\
\hline \multicolumn{3}{|l|}{ Neck dissection } \\
\hline No & 13 & 41 \\
\hline Unilateral & 6 & 19 \\
\hline Bilateral & 12 & 37 \\
\hline Information missing & 1 & 3 \\
\hline \multicolumn{3}{|l|}{ Postoperative (C)RT } \\
\hline No & 23 & 72 \\
\hline Yes & 9 & 28 \\
\hline \multicolumn{3}{|l|}{ Voice prosthesis } \\
\hline Provox 2 & 4 & 13 \\
\hline Provox Vega & 18 & 56 \\
\hline Provox ActiValve & 10 & 31 \\
\hline \multicolumn{3}{|l|}{ Patch } \\
\hline StabiliBase & 11 & 34 \\
\hline FlexiDerm & 10 & 31 \\
\hline OptiDerm & 5 & 16 \\
\hline XtraBase & 4 & 13 \\
\hline Other & 2 & 6 \\
\hline \multicolumn{3}{|l|}{$H M E$} \\
\hline Daily & 32 & 100 \\
\hline+ ASV use & 9 & 28 \\
\hline
\end{tabular}

Abbreviations: ASV, Automatic Speaking Valve; (C)RT, (chemo)radiotherapy; HME, Heat and Moisture Exchanger; TL, Total Laryngectomy; SD, standard deviation.

suitable for sensitive and/or breached skin and its baseplate is designed to also accommodate deep tracheostomas.

The SBO was compared with the OD in a feasibility study with a $2 \times 2$ crossover design. After inclusion, the patients 

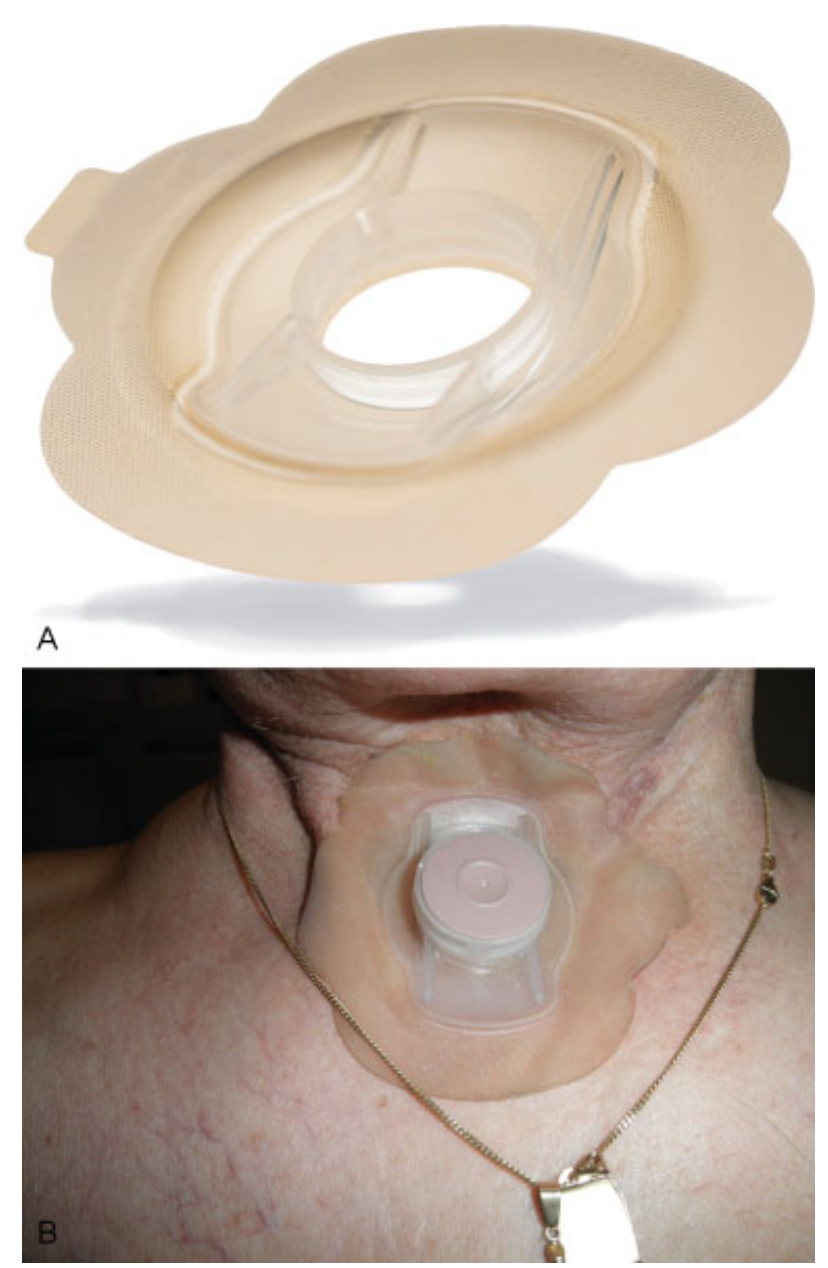

Fig. 1 StabiliBase OptiDerm (SBO); (A) a technical drawing of the SBO without liner (frontal view) showing the stable and conical base; (B) attached to a patient with the heat and moisture exchanger in situ.

consecutively used 5 OD and 5 SBO patches in the order assigned by randomization. The primary outcome measure was overall patient preference, based on the various aspects of the patch addressed in the study-specific questionnaires (see below). The secondary outcome parameters were: device life, patient satisfaction (skin irritation, comfort, voice/speech), ease of application, and quality of life. Study-specific structured questionnaires were completed at baseline, after the use of the first 5 patches and after the use of the following 5 patches. Questionnaires addressed skin irritation, ease of application, ease of removal, cleanliness, mucus collection, fit, comfort, use of other devices in combination with patch, appearance, voice quality, air leakage, adherence and cleaning tracheostomy/ voice prosthesis. Answers were reported on a four-level Likert scale. Patients rated satisfaction regarding device life and voice quality using a $10-\mathrm{cm}$ Numeric Rating Scale (NRS) $(0=$ worst and $10=$ best). Quality of life was assessed using the EuroQOL5 Dimension-5 Level questionnaire (Eq. 5D5L). ${ }^{13}$ The Eq. 5D5L is a validated instrument using scores in five health care dimensions (mobility, self-care, daily activities, pain/discomfort and anxiety/depression) and a $100-\mathrm{mm}$ visual analogue scale (VAS). During the study period, patients kept a diary to record the device life of each patch and the number of hours per day of HME use. At the end of the study patients were asked to complete a comparative questionnaire. Patients had to compare the SBO with the OD and also with their normally used patch if different from the OD.

The primary outcome of this study was patient preference. The goal was that $40 \%$ of the participants preferred the SBO to the $\mathrm{OD}, 5 \%$ considered the SBO to be worse, whereas the remainder considered both patches to be equally good (45\%) or bad (10\%). Based on earlier studies and given the assumption that in the absence of irritation, the duration of the seal is the deciding factor, this was a feasible goal to be expected and clinically relevant. ${ }^{9,10,12}$ A sample size of 30 pairs will have $82 \%$ of power to detect a difference in proportions of 0.350 , while the proportion of discordant pairs is expected to be 0.450 , using a sign test of equality of paired proportions with a 0.05 two-sided level of significance. As this was a short study and no risks have been associated with participation in the study, the dropout rate was expected to be $<5 \%$. Statistical analyses were conducted using the IBM Statistical Package for the Social Sicencessoftware (IBM Corp., Armonk, NY, US), version 22.0. Frequencies were explored using the Kolmogorov-Smirnov test. Parametrically distributed data are shown as mean \pm standard deviation and analyzed using the paired t-test. Non-parametrical data are presented as median (inter quartile range) and were analyzed using the Wilcoxon signed-rank test. The Likert Scales rendered ordinal data from three related samples. This data was analyzed using the Friedman test. If the groups differed significantly, a Wilcoxon signed-rank test was used to determine which groups were different. A $p$-value $<0.05$ was considered significant.

\section{Results}

The characteristics of the 32 patients enrolled in the study are shown in - Table 1. One patient withdrew from the study in the first week because of recurrent disease and was excluded from further analysis. Twenty-seven males and four females remained. Four patients did not use all of the study patches. Reasons were: skin irritation after using the $\mathrm{SBO}$, poor adherence of the SBO to the skin, poor adherence of the OD to the skin and painful skin after using the OD. An overview of completed questionnaires is shown in -Table 2 .

Table 2 Completed questionnaires

\begin{tabular}{|l|l|}
\hline Questionnaire & $\mathbf{n}$ \\
\hline Baseline & $31^{*}$ \\
\hline OD & $30^{* *}$ \\
\hline SBO & $30^{* * *}$ \\
\hline Comparative 'Normally used patch' - SBO & $25^{* * *} /^{* * * *}$ \\
\hline Comparative OD - SBO & $30^{* * *}$ \\
\hline
\end{tabular}

Abbreviations: OD, OptiDerm; SBO, StabiliBase OptiDerm. Notes: *One patient dropped out right after baseline data collection. These data were removed from the analysis.

${ }^{* *}$ One patient did not complete the OD questionnaire (poor adherence).

*** One patient did not complete the SBO questionnaire and the comparative questionnaires (poor adherence).

${ }^{* * * *}$ For the five patients who were already using $\mathrm{OD}$ at baseline, the OD-SBO comparative questionnaire was used as normally used patch-SBO comparative questionnaire. 
When patients compared the OD with the SBO, 12 of 30 patients $(40.0 \%)$ preferred the OD. Thirteen patients preferred the $\mathrm{SBO}(43.3 \%$ ) and 5 patients (16.7\%) expressed no preference. In comparison with their normally used patch, 18 patients (60.0\%) indicated a preference for the normally used patch, 7 patients (23.3\%) for the SBO and 5 patients (16.7\%) indicated no preference. Of the 5 patients who were using the OD at baseline (preference for OD 3 , for SBO 1 , no preference ${ }^{1}$ ), the answers to the comparative OD-SBO questionnaire were used as 'normally used patch-SBO-data' in these analyses (-Fig. 2).

Device life assessment was based on the data provided by the patients, who reported on at least 3 out of $5 \mathrm{OD} / \mathrm{SBO}$ patches. For the OD, the median device life was 18.5 hours ( $n=26$; range $0.5-109.9$ ) and for the SBO this was 19.6 hours ( $n=27$; range $0.5-163.0)(p=0.290)$. When data were split for patches used to apply an ASV or a HME, no significant differences were found between device life of the SBO and OD. There was an increase in device life in 15 out of 26 patients with the SBO compared with the OD, with a mean factor of 1.44. In 2 patients, there was no difference, and in 9 patients, there was a decrease of the device life with the SBO compared with the OD with a mean factor of 0.76 . The overall mean factor was 1.17 . The median self-reported device life in the 15 patients who had an increased device life with the SBO, was 14.47 hours (range 1.9-109.9) with the OD and 19.60 hours (range 2.35-163.01) with the SBO.

Analysis of fit, comfort, appearance, speech, air leakage and adherence, measured at baseline, after using 5 OD patches and after using 5 SBO patches, showed a statistically significantly better outcome for the normally used patch compared with the SBO and the OD. No significant differences regarding these variables were found between the SBO and OD.

With respect to skin irritation, no significant difference was found between the normally used patch, OD and SBO. When asked to compare these two patches, $17 \%$ experienced less skin irritation with the OD, 23\% experienced less skin irritation with the SBO and 60\% experienced no difference $(n=30)$. Compared with the normally used patch $(n=25)$, $12 \%$ experienced less skin irritation with that patch, $32 \%$ with the SBO and 56\% experienced no difference.

Participants indicated significantly less discomfort with their normally used patch compared with the SBO $(p=0.001, n=30)$. When asked to compare the normally used patch with the SBO, $52 \%$ found that patch more comfortable to wear, $24 \%$ found the SBO more comfortable and $24 \%$ found no difference. When asked to compare the OD and SBO, $33 \%$ had less discomfort with the SBO, $40 \%$ with the OD and $27 \%$ indicated no difference.

Overall voice and speech was measured using a NRS. There was no statistically significant difference between the normally used patch and the SBO. Only the OD received a statistically significantly lower score compared with the normally used patch ( $p=0.004, n=30$ ), and compared with the SBO $(p=0.007, n=30)$. Furthermore, no significant differences in applying the patch and in the quality of life (according to Eq. 5D5L) between the normally used patch, OD and SBO were found.

Finally, regarding future use, 15 out of 28 patients (53.6\%) reported that they would keep their normally used patch in the future. Of the 13 remaining patients answering this question, 6 (21.4\%) will use the OD, 5 (17.9\%) the SBO and $2(7.1 \%)$ a combination of the normally used patch with SBO. Data of two patients were missing. Those seven patients who will use the SBO or a combination of the normally used patch and SBO in the future consist of two former regular patch users (29\%), four SB users (57\%) and one tracheostomy button user (14\%).

During this study, six adverse device effects were registered. There were complaints about skin irritation, painful removal of the patch and poor adherence. All reports were expected effects of using a tracheostomy patch.
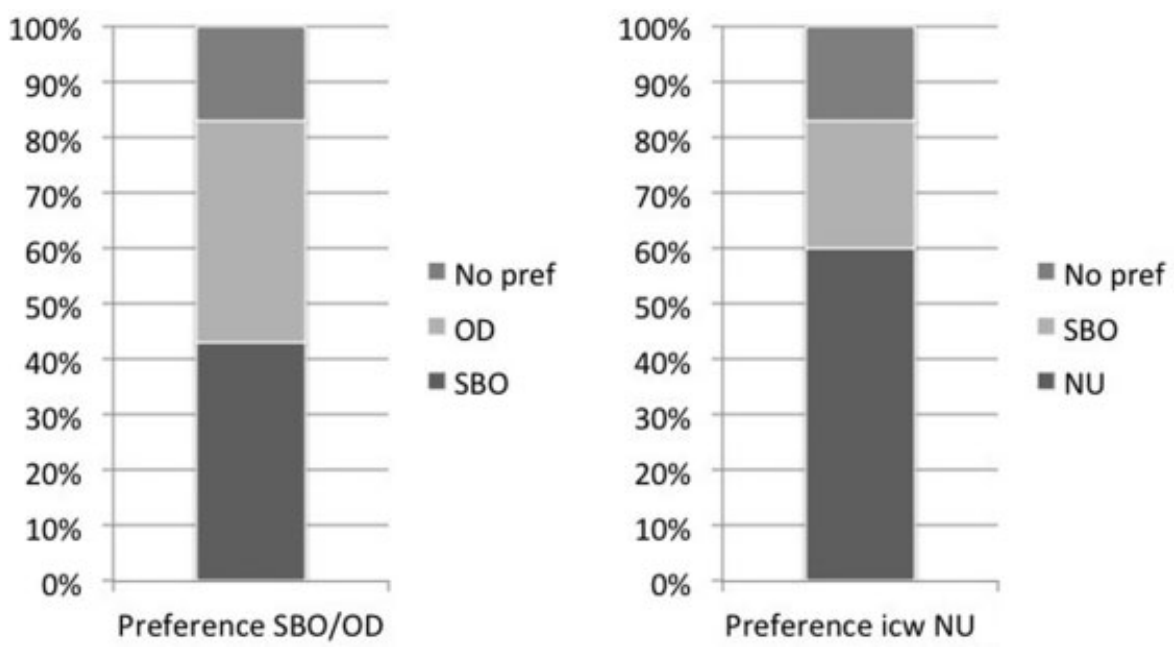

Fig. 2 To illustrate the added value of more patch choices, on the left, the preference at the end of the study for either of the 2 hydrocolloid patches (SBO = StabiliBase OptiDerm, $n=13 ; \mathrm{OD}=$ OptiDerm, $n=12$; No pref $=$ No preference for either of the two, $n=5$ ); on the right, the preference in comparison with (icw) the normally used patch ( $\mathrm{NU}=$ normally used patch, $n=18 ; \mathrm{SBO}, n=7 ;$ No pref. $n=5$ ). 


\section{Discussion}

This prospective clinical trial on the evaluation of the SBO, a new patch with stable conical base and hydrocolloid adhesive for peristomal attachment of postlaryngectomy pulmonary and voice rehabilitation, shows that this patch is a valuable addition to the variety of options needed to suit more laryngectomized patients.

With a quarter of the patients choosing the SBO, or a combination of the normally used patch with the SBO, it is clear that the SBO is suitable for a subgroup of patients. This subgroup might consist of patients who are using a SB as their main patch and would like to alternate with a more skin-friendly patch, keeping in mind that the median device life of the standard SB is roughly 1.8 times longer because of its stickier adhesive material. ${ }^{9}$ These patients may benefit when they prefer a stable base around the tracheostomy, but cannot use the SB (all day) due to sensitive/breached skin.

The results show that the device life of the SBO is not significantly increased compared with the OD (both hydrocolloid adhesives). However, for those 15 patients who had an increased device life with the SBO compared with the OD, the increase is clinically relevant. The difference ( 19.60 hours versus 14.47 hours) often made it possible for those patients to replace the patch only once per 24 hours. Nevertheless, a majority of the patients preferred the normally used patch, because in the absence of skin irritation, the duration of the seal is the decisive factor for their 'patch-choice'. As the mean interval between TL and participation in this study was 6.5 years, most patients have extensive experience with several peristomal attachment possibilities and found their optimal attachment modality. Still, there are patients (23.3\%) who prefer the SBO to their normally used patch. These results show there are still possibilities for further innovation, despite the wide range of patches already available to laryngectomized patients which is not surprising, given the wide variations in peristomal anatomy. ${ }^{14}$ So far, only a few clinical studies have been conducted to investigate peristomal patches. Because of the wide variety of rehabilitation options for laryngectomized patients, however, a good insight in patients' needs is necessary to find the optimal rehabilitation options. For instance, the study by Hilgers et al (2012) describes that there is no one-size-fits-all solution and emphasizes the need for a range of device options, which means that this new patch is a welcome development. ${ }^{9}$

In the present, relatively small study, although there was no selection based on the presence or absence of skin irritation, there still might have been some selection bias. For example, patients who were unable to use the SBO, such as patients with anatomical irregularities in the area of the patch that interfere with the stable base of the patch, were excluded. Furthermore, some variables that might influence device life were not collected. For example, we did not ask the patients to register hours of ASV use in their diaries and we did not measure tracheostomy dimensions and local anatomy, factors that obviously can influence the outcomes. ${ }^{8}$

The cost of these new patches was not a topic of this study. Although according to the manufacturer the periodical costs for various patches is quite comparable, to analyze costs in a meaningful way, a proper cost-effectiveness study would have been needed. This would require collecting additional data to those of a standard clinical study. Moreover, since costs and reimbursement systems vary widely between countries, even making vague suggestions about cost issues now would be speculative, at best. But this is certainly an interesting topic for studies in other countries.

\section{Conclusion}

Most patients preferred their normally used patch and SBO was favored by a subgroup. Therefore, SBO seems to be a valuable addition to the arsenal of devices already available and widens the options laryngectomized patients have for peristomal attachment of medical devices for pulmonary protection and rehabilitation.

\section{References}

1 Shelly MP, Lloyd GM, Park GR. A review of the mechanisms and methods of humidification of inspired gases. Intensive Care Med 1988;14(01):1-9

2 Parrilla C, Minni A, Bogaardt H, et al. Pulmonary Rehabilitation After Total Laryngectomy: A Multicenter Time-Series Clinical Trial Evaluating the Provox XtraHME in HME-Naïve Patients. Ann Otol Rhinol Laryngol 2015;124(09):706-713

3 Zuur JK, Muller SH, de Jongh FH, van Zandwijk N, Hilgers FJ. The physiological rationale of heat and moisture exchangers in postlaryngectomy pulmonary rehabilitation: a review. Eur Arch Otorhinolaryngol 2006;263(01):1-8

4 Hilgers FJ, Aaronson NK, Ackerstaff AH, Schouwenburg PF, van Zandwikj $\mathrm{N}$. The influence of a heat and moisture exchanger (HME) on the respiratory symptoms after total laryngectomy. Clin Otolaryngol Allied Sci 1991;16(02):152-156

5 Hilgers FJ, Ackerstaff AH, Van As CJ, Balm AJ, Van den Brekel MW, Tan IB. Development and clinical assessment of a heat and moisture exchanger with a multi-magnet automatic tracheostoma valve (Provox FreeHands HME) for vocal and pulmonary rehabilitation after total laryngectomy. Acta Otolaryngol 2003; 123(01):91-99

6 Op de Coul BM, Ackerstaff AH, van As-Brooks CJ, et al. Compliance, quality of life and quantitative voice quality aspects of hands-free speech. Acta Otolaryngol 2005;125(06):629-637

7 van der Houwen EB, van Kalkeren TA, Burgerhof JG, van der Laan $B F$, Verkerke GJ. In vitro evaluation of the iValve: a novel handsfree speech valve. Ann Otol Rhinol Laryngol 2011;120(12): 814-819

8 van der Houwen EB, van Kalkeren TA, Post WJ, Hilgers FJ, van der Laan BF, Verkerke GJ. Does the patch fit the stoma? A study on peristoma geometry and patch use in laryngectomized patients. Clin Otolaryngol 2011;36(03):235-241

9 Hilgers FJ, Dirven R, Wouters Y, Jacobi I, Marres HA, van den Brekel MW. A multicenter, prospective, clinical trial evaluating a novel adhesive baseplate (Provox StabiliBase) for peristomal attachment of postlaryngectomy pulmonary and voice rehabilitation devices. Laryngoscope 2012;122(11):2447-2453

10 Ackerstaff AH, Hilgers FJ, Balm AJ, Tan IB. Long-term compliance of laryngectomized patients with a specialized pulmonary rehabilitation device: Provox Stomafilter. Laryngoscope 1998;108(02): 257-260

11 Ackerstaff AH, Hilgers FJ, Aaronson NK, Balm AJ, van Zandwijk N. Improvements in respiratory and psychosocial functioning following total laryngectomy by the use of a heat and moisture exchanger. Ann Otol Rhinol Laryngol 1993;102(11):878-883 
12 Hilgers FJ, Ackerstaff AH, Balm AJ, Gregor RT. A new heat and moisture exchanger with speech valve (Provox stomafilter). Clin Otolaryngol Allied Sci 1996;21(05):414-418

13 Dolan P. Modeling valuations for EuroQol health states. Med Care 1997;35(11):1095-1108
14 Dirven R, Wouters Y, Vreeken R, Maal TJ, Marres HA. Threedimensional stereophotogrammetrical analysis of peristomal fixation of adhesive base plates during hands-free speech among laryngectomised patients related to tracheostoma volumes. Clin Otolaryngol 2012;37(02):124-129 